\title{
Systemic inflammation and oxidative stress contribute to acute kidney injury after transcatheter aortic valve implantation
}

\author{
Arunraj Navaratnarajah" ${ }^{1,2}$, Amit Bhan ${ }^{2}$, Emma Alcock $^{3}$, Tracy Dew ${ }^{2}$, Mark Monaghan $^{2}$, \\ Ajay M. Shah ${ }^{2}$, Olaf Wendler ${ }^{4}$, Philip MacCarthy ${ }^{2}$, Rafal Dworakowski ${ }^{2,5}$ \\ ${ }^{1}$ Renal Section, Department of Medicine, Hammersmith Hospital Campus, \\ Imperial College London, United Kingdom \\ ${ }^{2}$ Department of Cardiology, King's College Hospital and King's College London, \\ British Heart Foundation Centre, London, United Kingdom \\ ${ }^{3}$ Department of Anaesthesia, King's College Hospital and King's College London, \\ British Heart Foundation Centre, London, United Kingdom \\ ${ }^{4}$ Department of Cardiothoracic Surgery, King's College Hospital and King's College London, \\ British Heart Foundation Centre, London, United Kingdom \\ ${ }^{5}$ Department of Cardiology, Medical University of Gdansk, Poland
}

\begin{abstract}
Background: Acute kidney injury (AKI) is a frequent complication of transcatheter aortic valve implantation (TAVI) and has been linked to preexisting comorbidities, peri-procedural hypotension, and systemic inflammation. The extent of systemic inflammation after TAVI is not fully understood. Our aim was to characterize the inflammatory response after TAVI and evaluate its contribution to the mechanism of post-procedural AKI.

Methods: One hundred and five consecutive patients undergoing TAVI at our institution were includ$e d$. We analyzed the peri-procedural inflammatory and oxidative stress responses by measuring a range of biomarkers (including C-reactive protein [hsCRP], cytokine levels, and myeloperoxidase [MPO]), before TAVI and 6, 24, and 48 hours post-procedure. We correlated this with changes in renal function and patient and procedural characteristics.

Results: We observed a significant increase in plasma levels of pro-inflammatory cytokines (hsCRP, interleukin 6, tumor necrosis factor alpha receptors) and markers of oxidative stress (MPO) after TAVI. The inflammatory response was significantly greater after transapical (TA) TAVI compared to transfemoral (TF). This was associated with a higher incidence of AKI in the TA cohort compared to TF (44\% vs. 8\%, respectively, $p<0.0001)$. The incidence of AKI was significantly lower when $N$-acetylcysteine (NAC) was given peri-procedurally $(12 \%$ vs. $38 \%, p<0.005)$. In multivariate analysis, only the TA approach and no use of NAC before the procedure were independent predictors of AKI. Conclusions: TAVI creates a significant post-procedural inflammatory response, more so with the TA approach. Mechanisms of AKI after TAVI are complex. Inflammatory response, hypoperfusion, and oxidative stress may all play a part and are potential therapeutic targets to reduce/prevent AKI. (Cardiol J 2022; 29, 5: 824-835)
\end{abstract}

Key words: severe aortic stenosis, transcatheter aortic valve implantation, acute kidney injury, systemic inflammation

Address for correspondence: Dr. Rafal Dworakowski, MD, PhD, Consultant Cardiologist, King's College Hospital, Cardiovascular Division, Hambledon Wing, Denmark Hill, London, SE5 9RS, United Kingdom, tel: +44-(0)203 299 1308, fax: +44-(0)203 299 3489, e-mail: rdworakowski@nhs.net

Received: 3.09.2019 Accepted: 11.05.2020 Early publication date: 1.12.2020

This article is available in open access under Creative Common Attribution-Non-Commercial-No Derivatives 4.0 International (CC BY-NC-ND 4.0) license, allowing to download articles and share them with others as long as they credit the authors and the publisher, but without permission to change them in any way or use them commercially. 


\section{Introduction}

Acute kidney injury (AKI) is a recognized complication of conventional valve surgery using cardiopulmonary bypass and is associated with poor outcomes [1]. Aside from preexisting comorbidities, important contributors include hypotension during cardiopulmonary bypass and post-operative systemic inflammation. TAVI does not require cardiopulmonary bypass, but post-procedural AKI frequently occurs, in $19 \%$ to $33 \%$ of patients $[2,3]$. Although the prevalence of chronic kidney disease in transcatheter aortic valve implantation (TAVI) patients is higher than in surgical cohorts (because of their greater age and higher overall risk), the exact mechanism of AKI following TAVI is unclear. In some studies, AKI has been linked with the volume of intra-operative contrast agent used [4], but other factors have also been identified including blood transfusions [5].

Little is known about the magnitude of the inflammatory response, oxidative stress, and myocardial/renal injury after TAVI. Systemic inflammatory response syndrome (SIRS) after TAVI, measured using C-reactive protein (CRP) and body temperature, is an independent predictor of mortality [6]. The precise etiology of SIRS in this setting is unclear but may in part relate to myocardial damage and pump failure. It is clear that myocardial damage is a poor prognosticator, and Yong et al. [7] have shown recently that a peri-procedural increase in markers of myocardial injury independently predicts a poor outcome at 30 days after TAVI. Inflammatory markers such as interleukin 6 (IL-6) [8] and tumor necrosis factor alpha $(\mathrm{TNF} \alpha)$ [9] have been correlated with AKI after coronary artery bypass grafting, and although likely to be relevant, they have not previously been demonstrated in a TAVI population.

The aim of our study was to analyze and clarify the systemic inflammatory response, the change in oxidative stress, and magnitude of myocardial injury after TAVI and thereafter to explore associations with AKI and procedural outcome.

\section{Methods}

\section{Patient population}

We prospectively included 105 consecutive patients with severe aortic stenosis, who underwent TAVI either via a transfemoral (TF TAVI, $\mathrm{n}=60$ ) or a transapical approach (TA TAVI, $\mathrm{n}=45$ ) using Edwards-Sapien and Sapien XT transcatheter heart valves (Edwards Lifesciences, Inc., CA, USA) between July 2009 and July 2012. The study was approved by the local research Ethics Committee. All subjects provided written informed consent.

Mortality tracking was undertaken by the National Health Service Central Register using unique patient identifiers for all patients enrolled in this study.

The primary endpoint of the study was the occurrence of AKI. We utilized the Acute Kidney Injury Network classification of AKI [10]. Clinical outcome and complications were recorded using Valve Academic Research Consortium-2 definitions [11]. The estimated glomerular filtration rate was calculated by the simplified Modification of Diet in Renal Disease formula.

Systemic inflammatory response syndrome was defined as fulfilling at least two of the following criteria during the first $48 \mathrm{~h}$ after TAVI: temperature $<36.0^{\circ} \mathrm{C}$ or $>38.0^{\circ} \mathrm{C}$, heart rate $>90 \mathrm{bpm}$, and leucocyte count $>12$ or $<4\left(10^{9} / \mathrm{L}\right)$.

\section{Preoperative protocol to prevent AKI}

$\mathrm{N}$-acetylcysteine (NAC) was given at the physician's discretion 1 day pre-TAVI at $600 \mathrm{mg}$ bd and continued for $48 \mathrm{~h}$ post TAVI in $53 \%$ of patients $(n=60)$.

\section{TAVI procedure and anesthesia}

Transfemoral TAVI was performed using the conventional technique [12]. A similar general anesthetic technique was used in all cases regardless of access site. Surgical exposure of the femoral artery was used as the mode of arterial access in TF patients. Initial fluid replacement was with $1000 \mathrm{~mL}$ compound sodium lactate. Blood pressure was maintained (systolic $>100 \mathrm{mmHg}$ ) during the procedure with metaraminol boluses (50-100 $\mu \mathrm{g}$ ). No patient required catecholamine infusion post-procedure.

\section{Invasive cardiac output monitoring}

The FloTrac system uses a clinically validated algorithm to provide continuous cardiac output, stroke volume, and systemic vascular resistance measurements. Heart rate, and systolic and diastolic blood pressure were recorded at each time point. A Vigileo ${ }^{\mathrm{TM}}$ (Edwards Lifesciences, Irvine, CA, USA) monitor with software version 1.01 was connected to the radial artery catheter via a FloTrac ${ }^{\mathrm{TM}}$ (Edwards Lifesciences, Irvine, CA, USA) pressure sensor.

\section{Biomarkers}

Blood samples were obtained pre-TAVI and $4-6 \mathrm{~h}, 22-26 \mathrm{~h}$, and $44-52 \mathrm{~h}$ post-procedure from 
a central line. Intervals for sampling were based on data from Sinning et al. [13], in which an elevated leucocyte count and IL-6 was already observed $4 \mathrm{~h}$ after TAVI and reached a peak level at $48 \mathrm{~h}$ after TAVI. Serum was isolated within $1 \mathrm{~h}$ of collection and samples stored at $-80^{\circ} \mathrm{C}$ until thawed for determination of biomarkers, which were measured as follows:

Markers of inflammation and oxidative stress. Serum hsCRP was measured using a latex-enhanced immunoturbidimetric assay (PZ Corman, Lublin, Poland). The precision of the assays is expressed as the between-run coefficient of variation $(\% \mathrm{CV})$. The $\% \mathrm{CV}$ of the hsCRP assay for concentrations of $0.047,0.218$, and $0.976 \mathrm{mg} / \mathrm{L}$ was $6.97,3.34$, and $1.23 \%$, respectively.

Tumor necrosis factor alpha, tumor necrosis factor alpha receptor 1 (TNF $\alpha \mathrm{R} 1$ ), tumor necrosis factor alpha receptor 2 (TNF $\alpha$ R2), and IL-6 were measured using a Luminex ${ }^{\circledR}$ Bead-based Multiplex Assay system.

Serum myeloperoxidase (MPO) was measured using the quantitative sandwich enzyme-linked immunoassay (ELISA) technique (R\&D Systems Europe Ltd., United Kingdom). The \%CV of the MPO assay for concentrations of 15.7, 32.4, and $64.1 \mathrm{ng} / \mathrm{mL}$ was $7.5 \%, 7.7 \%$, and $6.6 \%$, respectively.

Markers of renal injury. Plasma neutrophil gelatinase-associated lipocalin (NGAL) was measured using a sandwich ELISA with wells coated with a monoclonal antibody against NGAL (BioPorto Diagnostics A/S, Denmark). The \%CV of the NGAL assay for concentrations $1.2-4 \mathrm{pg} / \mathrm{mL}$ was $3 \%$.

The assay is linear up to $1000 \mathrm{pg} / \mathrm{mL}$.

Serum cystatin $\mathrm{C}$ was measured using a latex-enhanced immunoturbidimetric assay (Siemens Healthcare Diagnostics Ltd., UK). The lowest concentration that can be distinguished from zero is $0.1 \mathrm{mg} / \mathrm{L}$.

Markers of myocardial injury. Serum creatine kinase MB (CK-MB) was measured using the Immulite 2000 assay (a chemiluminescent enzyme-labeled immunometric assay, reagent from Siemens Healthcare Diagnostics, Ltd., UK). The $\% \mathrm{CV}$ of the CK-MB assay for concentrations of $13.9,54.3$, and $95.4 \mathrm{ng} / \mathrm{mL}$ was $5.8 \%, 5.5 \%$, and $6.1 \%$, respectively.

Markers of heart failure and neurohormonal activation. $\mathrm{N}$-terminal pro-B-type natriuretic peptide (NT-proBNP) was measured on a Siemens Immulite 2000 analyzer (a two-site chemiluminescence immunoassay). The \% $\mathrm{CV}$ of the $\mathrm{BNP}$ assay for concentrations of $35.6,1430$, and $29,725 \mathrm{pg} / \mathrm{mL}$ was $5.4 \%, 3 \%$, and $4.1 \%$, respectively.
Receptor for interleukin-33 (ST2) (R\&D Systems Europe, Ltd., UK) was measured with a quantitative sandwich enzyme immunoassay technique. The \%CV of the ST2 assay for concentrations of 273,628 , and $1027 \mathrm{pg} / \mathrm{mL}$ was $5.6 \%, 4.4 \%$, and $4.5 \%$, respectively.

Aldosterone (Siemens Medical Solutions Diagnostics, 5700 West $96^{\text {th }}$ Street, LA, CA 90045-5597, USA) was measured with a solid-phase radioimmunoassay, based on aldosterone-specific antibody immobilized to the wall of a polypropylene tube. The $\% \mathrm{CV}$ of the aldosterone assay for concentrations of 65,448 , and $813 \mathrm{pg} / \mathrm{mL}$ was $3.5 \%, 15.3 \%$, and $19 \%$, respectively.

\section{Statistical analysis}

Data are presented as mean \pm standard deviation and \pm SEM when appropriate. Categorical variables are given as frequencies and percentages. The Fisher exact test was used to compare categorical variables in different groups. Continuous variables were tested for normality with the Shapiro-Wilk test. For continuous variables, Student's t-test or Kruskal-Wallis test, as appropriate, was performed for comparison between two groups. Two-way repeat measures ANOVA followed by Tukey post hoc test was used to compare groups at multiple time points. Variables with evidence of heterogeneity of variance were analyzed with nonparametric tests.

Logistic regression modeling was used to assess the short-term outcome and to determine independent predictors of AKI. In a multivariate regression analysis, we adjusted for significant predictors of 12 -month mortality in the univariate analysis. Survival curves according to the occurrence of AKI were plotted by the Kaplan-Meier method and compared using the Wilcoxon test. We employed a univariate Cox proportional hazard model to examine the association of AKI and other clinical characteristics with cumulative 12 -month mortality and to evaluate the impact on long-term clinical outcome. $\mathrm{P}<0.05$ was considered to be statistically significant.

Analyses were performed using the JMP 9 statistical package (SAS, USA).

\section{Results}

\section{Pre-procedural patient characteristics}

Baseline patient characteristics and use of pre-procedural medications with potential effect on post-operative kidney function are summarized in Table 1. AKI was associated with peripheral vascu- 
Table 1. Pre-procedural patient characteristics and medications according to the occurrence of acute kidney injury (AKI).

\begin{tabular}{|c|c|c|c|c|}
\hline & $\begin{array}{l}\text { All patients } \\
(n=105)\end{array}$ & $\begin{array}{l}\text { No AKI } \\
(n=81)\end{array}$ & $\begin{array}{c}\text { AKI } \\
(n=24)\end{array}$ & $\mathbf{P}$ \\
\hline \multicolumn{5}{|l|}{ Pre-procedural patient characteristics } \\
\hline Age [years] & $84 \pm 6$ & $84 \pm 6$ & $83 \pm 5$ & 0.8 \\
\hline Male gender & $50(48 \%)$ & $38(47 \%)$ & $12(50 \%)$ & 0.8 \\
\hline logistic EuroSCORE [\%] & $23 \pm 11$ & $22 \pm 10$ & $26 \pm 12$ & 0.2 \\
\hline Coronary artery disease & $51(49 \%)$ & $39(48 \%)$ & $12(50 \%)$ & 0.5 \\
\hline Peripheral vascular disease & $18(17 \%)$ & $9(11 \%)$ & $9(38 \%)$ & $<0.005$ \\
\hline Previous MI & $13(12 \%)$ & $8(10 \%)$ & $5(20 \%)$ & 0.2 \\
\hline Previous cardiac surgery & $22(21 \%)$ & $15(19 \%)$ & $7(29 \%)$ & 0.3 \\
\hline Previous $\mathrm{PCl}$ & $19(18 \%)$ & $12(15 \%)$ & $7(29 \%)$ & 0.1 \\
\hline Previous stroke/TIA & $15(15 \%)$ & $9(11 \%)$ & $6(25 \%)$ & 0.1 \\
\hline Chronic obstructive pulmonary disease & $32(31 \%)$ & $27(33 \%)$ & $5(21 \%)$ & 0.23 \\
\hline Pulmonary hypertension (> $60 \mathrm{mmHg}$ ) & $16(15 \%)$ & $11(13 \%)$ & $5(21 \%)$ & 0.7 \\
\hline Diabetes & $25(24 \%)$ & $17(21 \%)$ & $8(33 \%)$ & 0.2 \\
\hline Baseline hemoglobin [g/dL] & $12.4 \pm 1.6$ & $12.5 \pm 0.2$ & $11.8 \pm 0.3$ & 0.2 \\
\hline Creatinine $[\mu \mathrm{mol} / \mathrm{L}]$ & $94 \pm 28$ & $91 \pm 3$ & $105 \pm 6$ & $<0.05$ \\
\hline eGFR $\left[\mathrm{mL} / \mathrm{min} / 1.73 \mathrm{~m}^{2}\right]$ & $61 \pm 16$ & $63 \pm 2$ & $56 \pm 3$ & 0.05 \\
\hline LVEF [\%] & $53 \pm 12$ & $53 \pm 1$ & $53 \pm 3$ & 0.93 \\
\hline AVA $\left[\mathrm{cm}^{2}\right]$ & $0.68 \pm 0.18$ & $0.69 \pm 0.02$ & $0.69 \pm 0.02$ & 0.8 \\
\hline Peak pressure gradient [mmHg] & $80 \pm 25$ & $83 \pm 3$ & $77 \pm 5$ & 0.3 \\
\hline Mean pressure gradient $[\mathrm{mmHg}]$ & $46 \pm 16$ & $48 \pm 2$ & $42 \pm 3$ & 0.1 \\
\hline Left ventricle mass $[\mathrm{g}]$ & $171 \pm 51$ & $171 \pm 6$ & $167 \pm 11$ & 0.5 \\
\hline Cystatin C [mg/L] & $1.09 \pm 0.51$ & $1.04 \pm 0.33$ & $1.13 \pm 0.37$ & 0.3 \\
\hline NT-proBNP [pg/mL] & $4618 \pm 5934$ & $4513 \pm 752$ & $4962 \pm 908$ & 0.8 \\
\hline $\mathrm{NGAL}[\mu \mathrm{g} / \mathrm{L}]$ & $116.4 \pm 65.0$ & $108.8 \pm 6.5$ & $141.3 \pm 18.4$ & $<0.05$ \\
\hline \multicolumn{5}{|l|}{ Pre-procedural medications } \\
\hline Pre-procedural NAC & $60(57 \%)$ & $53(65 \%)$ & $7(29 \%)$ & $<0.005$ \\
\hline Thiazides & $9(9 \%)$ & $5(6 \%)$ & $4(17 \%)$ & 0.2 \\
\hline NSAIDs & $60(58 \%)$ & $46(58 \%)$ & $14(58 \%)$ & 1.0 \\
\hline Loop diuretics & $61(59 \%)$ & $47(59 \%)$ & $14(58 \%)$ & 1.0 \\
\hline ACEI/ARB & $45(44 \%)$ & $34(43 \%)$ & $11(46 \%)$ & 0.8 \\
\hline
\end{tabular}

$\mathrm{MI}$ - myocardial infarction; $\mathrm{PCl}$ - percutaneous coronary intervention; TIA — transient ischemic attack; eGFR — estimated glomerular filtration rate; LVEF — left ventricular ejection fraction; AVA — aortic valve area; NT-proBNP — N-terminal pro-B-type natriuretic peptide; NGAL neutrophil gelatinase-associated lipocalin; NAC - N-acetylcysteine; NSAIDs - non-steroidal anti-inflammatory drugs; ACEI — angiotensin converting enzyme inhibitors; ARB — angiotensin II receptor blockers

lar disease, higher pre-procedural creatinine, lower transvalvular gradients, and higher N-GAL levels. Only $29 \%$ of patients subsequently developing AKI had received NAC compared to $65 \%$ of those who did not develop AKI ( $p<0.005)$. Forty-seven per cent $(\mathrm{n}=21)$ of patients undergoing TA TAVI and $65 \%(\mathrm{n}=39)$ undergoing TF TAVI received NAC. There was a higher rate (RR 5.7; 1.6-25.1, $\mathrm{p}<0.05$ ) of AKI in patients who did not receive NAC (38\% vs. $12 \%, \mathrm{p}<0.005)$. This difference was significant in the TF group (19\% vs. $3 \%, \mathrm{p}<0.05)$ but not in the TA group (54\% vs. $29 \%, \mathrm{p}=0.08)$.

\section{Procedural outcome and predictors of post-TAVI AKI}

The valve prosthesis was successfully deployed in all patients $(100 \%)$ with no peri-procedural deaths $(0 \%)$ and no conversion to open surgery $(0 \%)$. The combined early safety endpoint at 30 days was $31 \%(\mathrm{n}=33)$. 

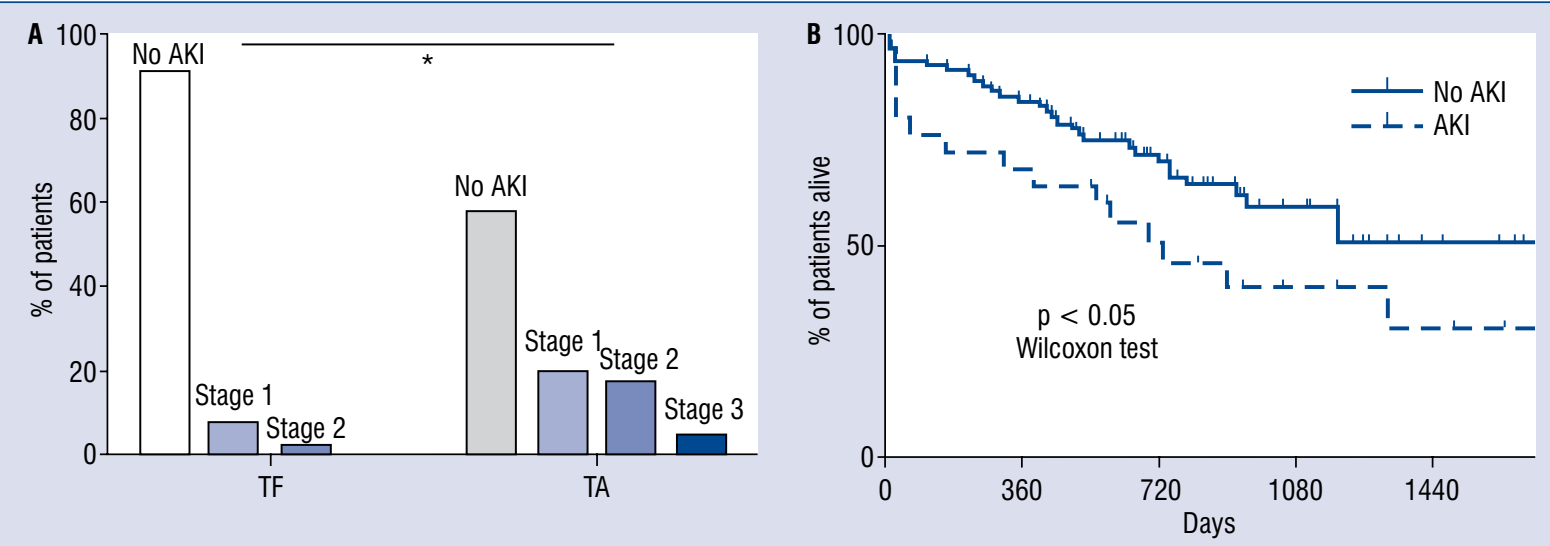

Figure 1. A. Incidence of acute kidney injury (AKI) according to access site (transfemoral [TF] vs. transapical [TA]). There was a significantly higher frequency of AKI in the transapical group (chi-square test, $\mathrm{p}<0.005$ ); B. Kaplan-Meier curves for patient with AKI and with no post-procedural AKI (Wilcoxon test, $p<0.05$ ). There was higher mortality in the group developing $\mathrm{AKI} ;{ }^{*} \mathrm{p}<0.005$.

Table 2. Procedural characteristics and post-procedural complications (as defined by the Valve Academic Research Consortium-2) according to the occurrence of acute kidney injury (AKI)

\begin{tabular}{lcccc}
\hline & $\begin{array}{c}\text { All patients } \\
(\mathbf{n}=105)\end{array}$ & $\begin{array}{c}\text { No AKI } \\
(\mathbf{n}=81)\end{array}$ & $\begin{array}{c}\text { AKI } \\
(\mathbf{n}=24)\end{array}$ \\
\hline Procedural characteristics & & & & P \\
Route transapical & $45(43 \%)$ & $26(35 \%)$ & $19(79 \%)$ & $<0.001$ \\
Valve size 23/26/29 mm & $55 / 41 / 9$ & $47 / 28 / 6$ & $8 / 13 / 3$ & 0.1 \\
Volume of contrast [mL] & $119 \pm 52$ & $117 \pm 5$ & $125 \pm 13$ & 0.6 \\
Blood transfusion required & $39(37 \%)$ & $28(35 \%)$ & $11(46 \%)$ & 0.3 \\
Pacing time [s] & $57 \pm 37$ & $54 \pm 4$ & $64 \pm 10$ & 0.4 \\
Post-procedural complications & & & 0.3 \\
Myocardial infarction & $0(0 \%)$ & $0(0 \%)$ & $0(0 \%)$ & $\mathrm{NS}$ \\
Stroke & $1(1 \%)$ & $1(1.2 \%)$ & $0(0 \%)$ & 1.0 \\
Major/life-threatening bleeding & $20(19 \%)$ & $16(20 \%)$ & $4(17 \%)$ & 1.0 \\
Major vascular/apical complications & $2(2 \%)$ & $1(1.2 \%)$ & $1(4.2 \%)$ & 0.4 \\
New conduction abnormality & $4(3.8 \%)$ & $2(2.5 \%)$ & $2(8.3 \%)$ & 0.2 \\
30-days mortality & $8(7.6 \%)$ & $5(6.2 \%)$ & $3(12 . \%)$ \\
Post-procedure aortic regurgitation $(\geq$ moderate) & $2(2 \%)$ & $0(0 \%)$ & $2(8.7 \%)$ & 0.4 \\
Device success & $102(96 \%)$ & $81(100 \%)$ & $21(87 \%)$ & $<0.05$ \\
\hline
\end{tabular}

Acute kidney injury occurred more often in TA patients $(44 \%, \mathrm{n}=20$, TF: $8 \%, \mathrm{n}=5 ; \mathrm{p}<0.0001)$ (Fig. 1A). There was no significant difference in baseline creatinine between the TF and TA groups (99 \pm 4 vs. $90 \pm 4 \mathrm{umol} / \mathrm{L}, \mathrm{NS})$, and there was no difference in baseline estimated glomerular filtration rate ( $62 \pm 2$ vs. $59 \pm 2 \mathrm{~mL} / \mathrm{min}, \mathrm{NS})$, hemoglobin $(12.4 \pm 0.2$ vs. $12.2 \pm 0.2 \mathrm{~g} / \mathrm{dL}, \mathrm{NS})$, or volume of contrast used during the procedure $(109 \pm 9$ vs. $126 \pm 5 \mathrm{~mL}, \mathrm{NS}$, TA vs. TF, respectively). There was a significant difference in total rapid pacing time, with longer rapid pacing in the TA group (TF $44 \pm$ \pm 3 vs. TA $75 \pm 8 \mathrm{~s}, \mathrm{p}<0.0005)$.

Procedural characteristics and post-procedural complications are summarized in Table 2.

In multivariate analysis TA TAVI and absence of NAC use pre-procedure were independent predictors of AKI (Table 3).

There was no difference in 30-day mortality between patients with post-procedural AKI and 
Table 3. Multivariate logistic regression analysis for predictors of acute kidney injury after transcatheter aortic valve implantation.

\begin{tabular}{lccc}
\hline Variables & OR & $95 \% \mathrm{Cl}$ & $\mathbf{P}$ \\
\hline NGAL & 0.99 & $0.98-1.01$ & 0.24 \\
Creatinine & 0.99 & $0.97-1.01$ & 0.37 \\
$\begin{array}{l}\text { Peripheral vascular } \\
\text { disease }\end{array}$ & 2.4 & $0.5-11.9$ & 0.28 \\
TA TAVI & 7.0 & $2.0-29.9$ & 0.002 \\
No NAC pre-procedure & 4.7 & $1.4-18.3$ & 0.01 \\
\hline
\end{tabular}

$\mathrm{Cl}$ - confidence interval; NAC - N-acetylcysteine; NGAL - neutrophil gelatinase-associated lipocalin; OR — odds ratio; TA TAVI transapical transcatheter aortic valve implantation

without ( $12.5 \%$ vs. $6.2 \%$, respectively, $\mathrm{p}=0.4)$, but there was a significant difference in overall mortality (Fig. 1B).

\section{Characterization of inflammatory response}

Inflammation and oxidative stress. TAVI was associated with a rise in pro-inflammatory cytokines including hsCRP, and TNF $\alpha$ and its receptors (R1 and R2). The magnitude of inflammation was associated with the severity of postprocedural AKI, especially at $44-52 \mathrm{~h}$ after the procedure (Fig. 2A, B). The inflammatory response was significantly greater after TA than after TF TAVI (Fig. 3A, D, E, F).

Myeloperoxidase levels rose significantly after TAVI from $540 \mathrm{ng} / \mathrm{L}$ at baseline to $992 \mathrm{ng} / \mathrm{L}$ at
$4-6 \mathrm{~h}$, to $1222 \mathrm{ng} / \mathrm{L}$ at $22-26 \mathrm{~h}$, and to $1412 \mathrm{ng} / \mathrm{L}$ at 44-52 h (MANOVA, $\mathrm{p}<0.0001$ ), suggesting an increase in oxidative stress. Again, these changes were more significant in stages II/III of AKI (Fig. 2B) and after TA TAVI (Fig. 3B).

Interleukin 6 increased significantly after TAVI but to a greater extent in the AKI group (Fig. 4B).

Systemic inflammatory response syndrome was observed in $8(8 \%)$ patients in the TA group (TA $8 \%$ vs. TF $0 \%, p<0.0005$ ), and of these, $50 \%$ had AKI (NS).

Markers of renal injury. Baseline NGAL was significantly higher in the AKI group (Table 1). NGAL levels increased post-procedure in all patients (baseline $115 \pm 7 \mathrm{pg} / \mathrm{mL}$ vs. $24 \mathrm{~h} 194 \pm$ $\pm 11 \mathrm{pg} / \mathrm{mL}, \mathrm{p}<0.0001)$, with a greater increase in patients who developed AKI (mean difference, respectively: 67 vs. $11,711 \mathrm{pg} / \mathrm{mL}, \mathrm{p}<0.005$, Fig. 4A).

Cystatin C levels rose significantly and were significantly higher at $44-52 \mathrm{~h}$ after TAVI in the group developing AKI (1.18 \pm 0.5 vs. $1.71 \pm 0.15$, $\mathrm{p}<0.005)$.

Markers of myocardial injury. There was no significant difference in myocardial injury between patients with and without AKI (CK-MB: $20 \pm 1.7$ vs. $23 \pm 14$, respectively, NS).

Markers of heart failure and neurohormonal activation. We observed a significant increase in ST2 and NT-proBNP after TAVI but with greater

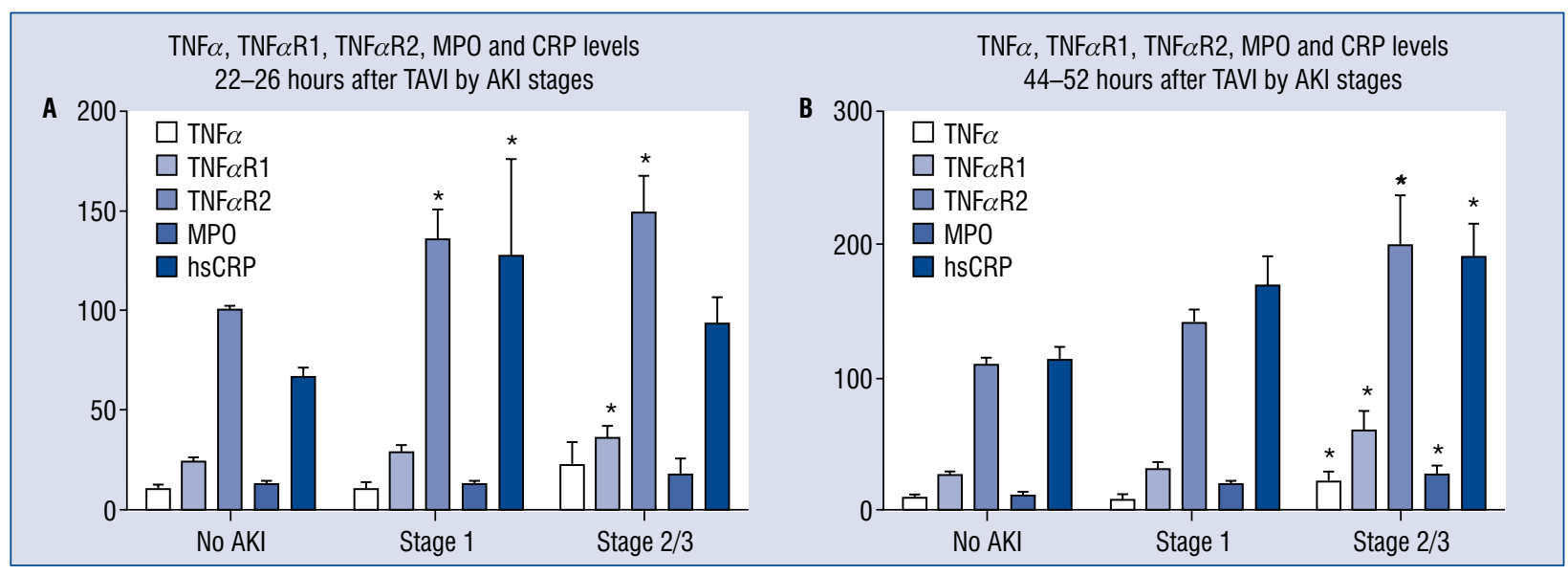

Figure 2. Levels of inflammatory markers and oxidative stress after transcatheter aortic valve implantation (TAVI) by stage of acute kidney injury (AKI); A. 22-26 h after TAVI; B. 44-52 h after TAVI. One-way ANOVA, followed by Tukey post-hoc test performed if ANOVA was significant, ${ }^{*} p<0.05$ from post hoc test significant difference against group with no postprocedural AKI. The magnitude of inflammatory reaction measured by levels of tumor necrosis factor alpha (TNF $\alpha$ ) and its receptors, myeloperoxidase (MPO) and C-reactive protein (CRP) 22-26 h after TAVI was higher in all patients who developed AKI regardless of its severity. On the other hand, 44-52 h after TAVI inflammatory markers remained high, but only in patients who developed AKI stage $2 / 3$. 


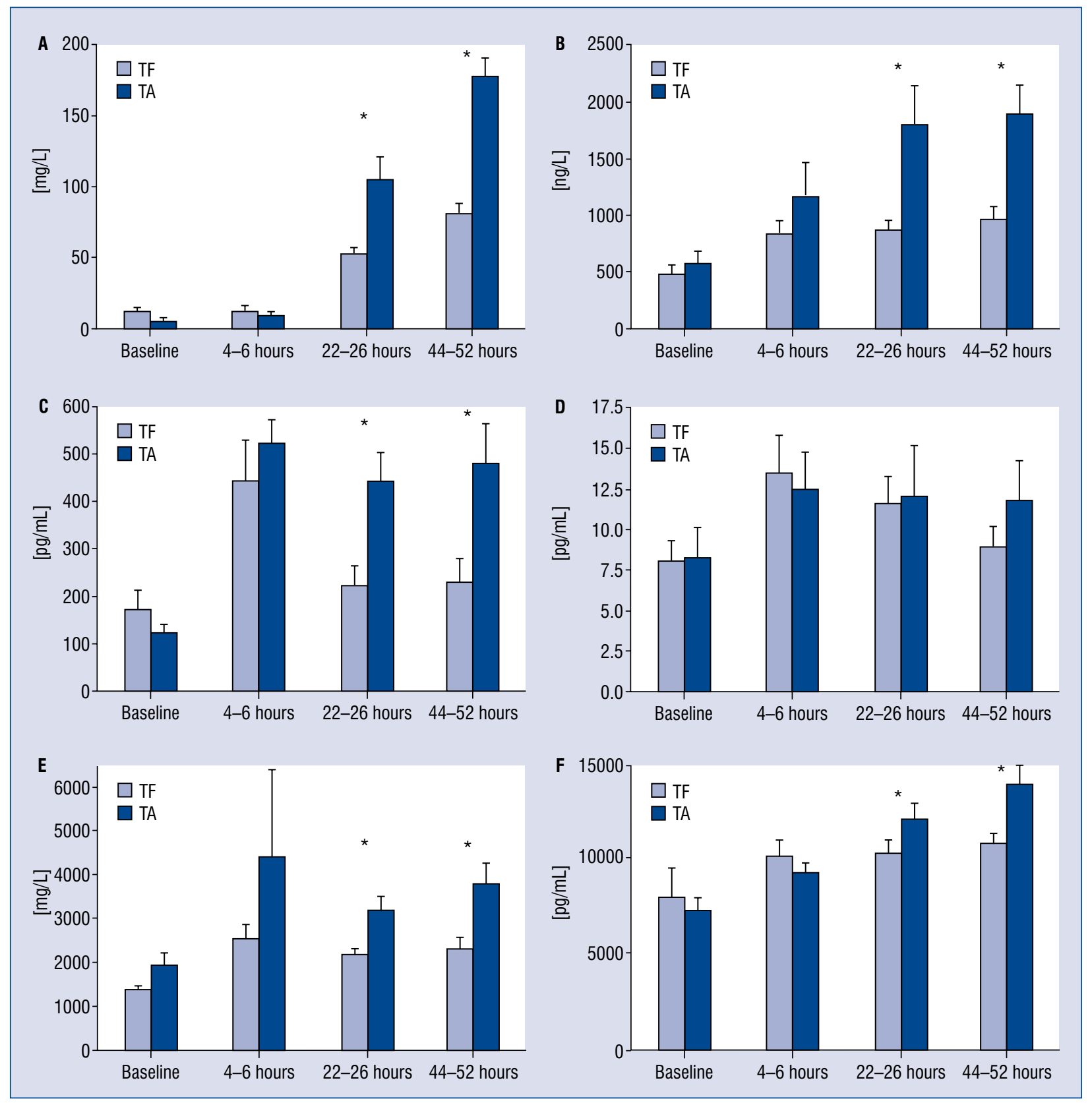

Figure 3. Changes in inflammatory markers and oxidative stress after transfemoral (TF) and transapical (TA) group 4-6 h, 22-26 h, and 44-52 h post-procedure. A. High-sensitivity C-reactive protein (hsCRP); B. Myeloperoxidase (MPO); C. Aldosterone; D. Tumor necrosis factor alpha (TNF $\alpha$ ); E. TNF $\alpha$ R1; F. TNF $\alpha$ R2. Two-way ANOVA followed by Bonferroni post hoc test, ${ }^{*} \mathrm{p}<0.05$. Levels of hsCRP, MPO, aldosterone, and TNF $\alpha \mathrm{R} 1$ and TNF $\alpha 2$ were significantly higher after TA transcatheter aortic valve implantation.

changes in the AKI group (Fig. 4C, D). There was no difference in aldosterone levels in the AKI group (not shown), but aldosterone was significantly higher after TA TAVI compared to TF TAVI (Fig. 3C).

Hemodynamic changes. No significant difference was observed in the first $24 \mathrm{~h}$ in heart rate, cardiac output, systolic blood pressure, diastolic blood pressure, and mean blood pressure in either group. Systemic vascular resistance was significantly lower at baseline and remained lower in patients who developed AKI (Fig. 5).

\section{Discussion}

This is the first study to characterize the inflammatory response associated with TAVI and 


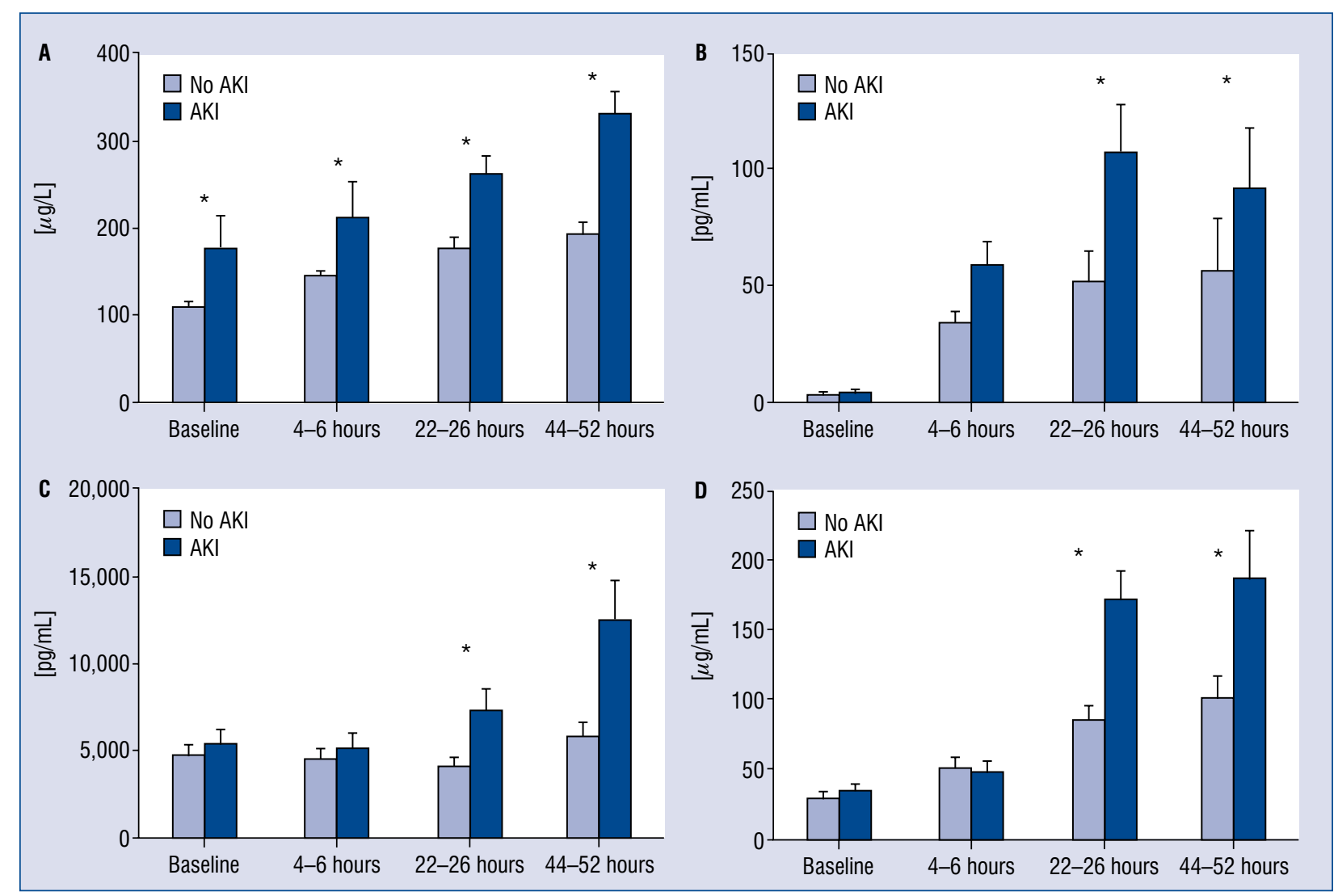

Figure 4. Changes in neurohormonal activation in acute kidney injury (AKI) and no AKI group 4-6 h, 22-26 h, and 44-52 h post-procedure. A. Neutrophil gelatinase-associated lipocalin (NGAL); B. Interleukin 6; C. N-terminal pro-B-type natriuretic peptide (NT-proBNP), D. Receptor for interleukin 33 (ST2); Two-way ANOVA followed by Bonferroni post hoc test, ${ }^{*} p<0.05$. NGAL levels were higher pre-transcatheter aortic valve implantation (TAVI) and were rising after the procedure significantly more in the AKI group. Interleukin 6 levels increase after TAVI from 4-6 h continuing rise over next hours. The rise in interleukin 6 levels was significantly higher in the AKI group at 22-26 h and 44-52 h. BNP and ST2 levels were rising significantly only in the AKI group at 22-26 h and 44-52 h.

explore its relationship with post-procedural AKI. We have identified pre- and peri-operative risk factors for developing AKI, including peripheral vascular disease, TA approach, and post-TAVI paravalvular aortic regurgitation. Furthermore, there is an association between the risk of AKI and the magnitude of the inflammatory response.

On multivariate analysis, pre-TAVI renal impairment was not associated with post-procedural AKI. Although not powered to show this definitively, our results are in concordance with previously published data [14] from a cohort of 642 patients, divided according to pre-procedural renal function, in which, similarly, pre-procedural renal function was not a predictor of post-procedural AKI. This suggests that factors other than pre-existing renal function contribute to the development of AKI [15].

The association between inflammatory response and AKI after TAVI has been reported in another small study [16], with suggestion of less inflammation with the TF compared with the TA approach [17]. We too observed a greater increase in inflammatory markers and oxidative stress after TA compared with TF TAVI, and that TA TAVI was associated with a significantly greater rate of AKI. Consistent with this, we report a significant increase in pro-inflammatory cytokines (including IL- 6 and TNF $\alpha$ receptors), which are involved in the acute phase of the inflammatory response. These pro-inflammatory cytokines activate hepatic synthesis of acutephase proteins including CRP, which were also significantly increased in our study. This inflammatory reaction has been noted to correlate with AKI and multi-organ failure in other medical settings [8]. Furthermore, the inflammatory response provokes an increase in oxidative stress, which is a known cause of AKI. 


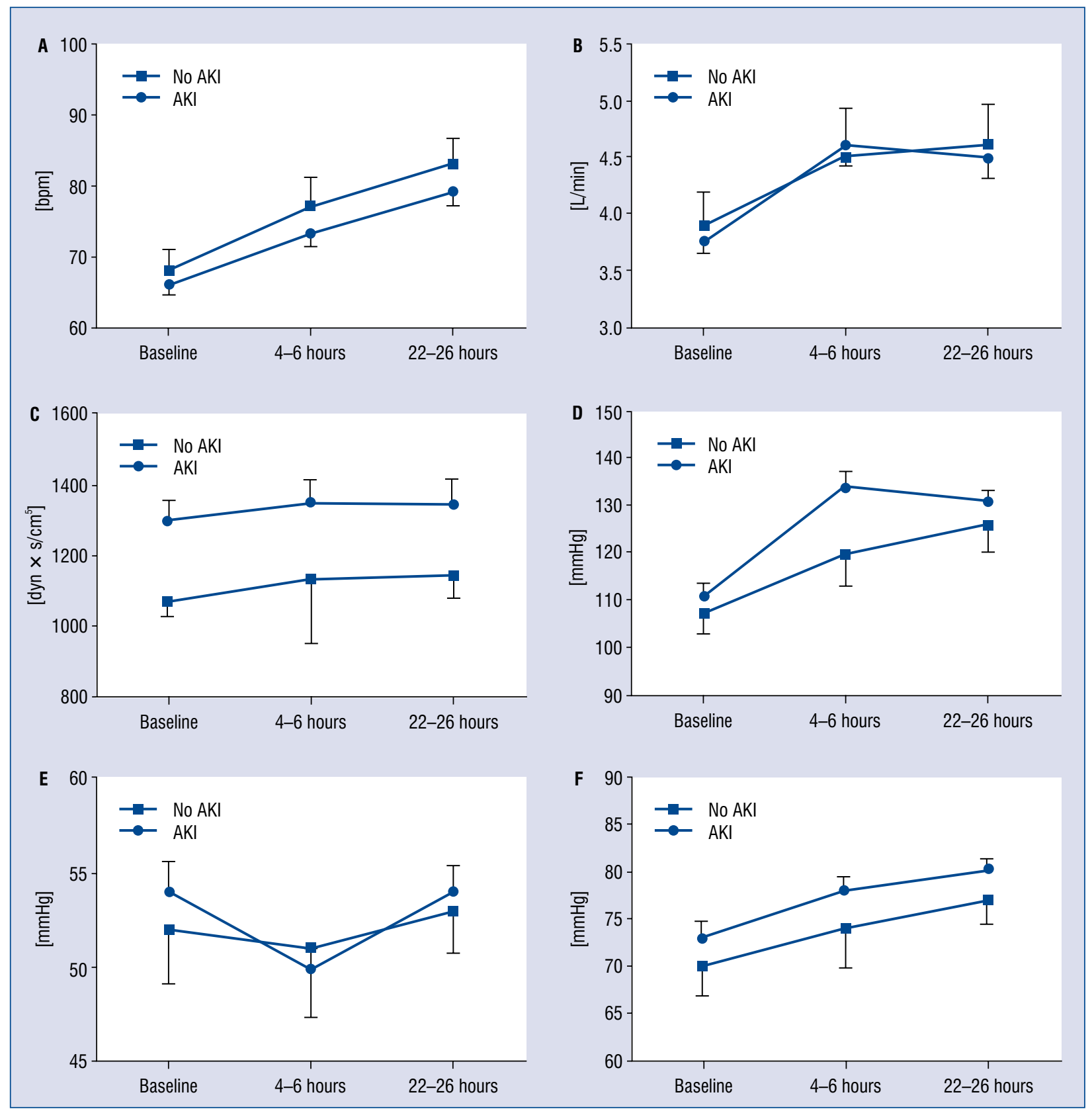

Figure 5. Changes in basic hemodynamics in acute kidney injury (AKI) and no AKI group 4-6 h and 22-26 h post-procedure; A. Heart rate (HR); B. Cardiac output (CO); C. Systemic vascular resistance (SVR); D. Systolic blood pressure (SBP); E. Diastolic blood pressure (DBP); F. Mean blood pressure (MBP). No significant difference was observed in the first $24 \mathrm{~h}$ in HR, CO, SBP, DBP, and MBP in either group. The SVR was significantly lower at baseline and remained lower in patients who developed AKI.

Sinning et al. [13] demonstrated that $46 \%$ of patients with SIRS develop AKI after TF TAVI, and we similarly found that $50 \%$ of patients with SIRS developed AKI. However, SIRS accounted only for $17 \%$ of all patients who developed AKI, confirming that lesser inflammatory responses and other factors are clearly important.
Neutrophil gelatinase-associated lipocalin, often labelled a "renal troponin", is a powerful predictor of AKI after onset of cell stressors such as ischemia-reperfusion or inflammation [18]. In the Translational Research Investigating Biomarker Endpoints in AKI study (TRIBE-AKI) elevated NGAL plasma levels predicted AKI and improved 
risk stratification [1]. In our cohort, NGAL levels were significantly increased after TAVI, and levels were greater in the TA group. This suggests that significant renal ischemia-reperfusion injury is probably implicated in the development of AKI. We were not able to predict AKI based on measurements of NGAL levels $4-6 \mathrm{~h}$ post-TAVI. Interestingly, levels of NGAL were higher at baseline in patients who went on to develop AKI, suggesting a possible predictive value in this setting.

Hemodynamic disturbance may also play a part in renal injury during TAVI. Procedural characteristics such as temporary hypotension during rapid pacing or balloon predilatation, or rhythm disturbances due to aberrant conduction or inotropic support, may be relevant. We, however, found no significant differences in major hemodynamic parameters between the group that developed AKI and those who did not, with no difference in duration of pacing nor size of valve prosthesis. Frequency of rapid pacing, type of prosthesis, and number of attempts to deploy a prosthesis were not examined but may be of interest. We have previously shown that left ventricular (LV) systolic function is transiently impaired post-TAVI, and this phenomenon may well be greater after a TA procedure [12]. Certainly, a longer rapid pacing time in the TA group (utilized by our surgeons for apical closure) can lead to myocardial stunning and transient depression of LV function [12]. More prolonged peri-procedural hypotension with subsequent myocardial damage in addition to myocardial injury related to direct apical access may both serve to activate the inflammatory response, which may explain excess AKI in the TA group.

Using CK-MB, we found no significant difference in myocardial injury between patients with and without AKI. The findings may have been different using troponin, a more specific biomarker for myocardial damage. Koskinas et al. [19] demonstrated the impact of post-procedural cardiac troponin elevation on both 30-day and 2-year prognosis. Mechanisms of troponin release during TAVI are, however, multifactorial, and as well as myocardial injury, they may reflect the complexity of underlying coronary artery disease, concentric LV hypertrophy, peri-procedural particle embolization into the coronary circulation, and pre-existing renal dysfunction. Peripheral vascular disease was more common in the AKI group. A useful extension to the current study would be to include measurements of embolization such as carotid flow and transcranial Doppler to determine the contribution of peripheral embolization in the development of AKI.
B-type natriuretic peptide (BNP) has been shown to be a good predictor of AKI after other forms of cardiac surgery [20] and a general predictor of outcome in patients with AKI [21]. Consistent with previously published data [22] we noticed a significant increase in BNP after TAVI with a greater BNP rise in patients developing AKI. Elevations of BNP may primarily reflect AKI instead of LV dysfunction in this setting. Increased intravascular volume stimulating cardiac secretion and reduced renal clearance of peptides serving as key mechanisms [23-25]. ST2, a member of the interleukin-1 receptor superfamily, was identified as a potential novel biomarker in patients with acute heart failure [26]. ST2 is up-regulated in isolated cardiomyocytes exposed to mechanical strain, and its levels correlate well with acute LV dysfunction independently of BNP $[27,28]$. We have previously shown that there is a significant increase in ST2 levels after successful TAVI, perhaps in response to peri-procedural myocardial dysfunction [12]. In the current study we observed a significant increase in ST2 levels in the AKI group, and after TA TAVI more than after TF TAVI. Like BNP, ST2 (by blocking the effects of interleukin-33) has been linked with AKI in other settings [29].

Another important contributor to post-procedural renal ischemia is moderate-severe post-TAVI paravalvular aortic regurgitation, which has previously been linked with AKI and severity associated with higher levels of BNP and troponin [6]. It is now widely accepted that para-prosthetic aortic regurgitation is associated with increased in-hospital and mid-term mortality following TAVI [30-32].

It has been suggested that post-procedural AKI is related to the amount of radio-opaque contrast used during the procedure [4, 14]. This hypothesis was not supported by Kong et al. [16], nor by our data. We did not see any link between volume of contrast used during TAVI and AKI, although all contrast volumes used were low. The volume of contrast was slightly higher in the TF TAVI group, which had a lower risk of AKI.

Tepel et al. [33] reported that NAC may prevent acute renal dysfunction by antioxidant action. NAC acts indirectly by boosting endogenous levels of the major cellular antioxidant, glutathione $[34,35]$. Despite proven efficacy in experimental models [36], the use of NAC in preventing AKI after cardiac surgery and coronary angiography in clinical practice remains controversial [37]. There are no data about the effects of NAC on AKI after TAVI. We incidentally observed that patients not given NAC pre TAVI had a higher incidence of 
post-procedural AKI, independent of access site and volume of contrast used. This may be influenced by unmeasured confounding factors including the appropriate clinical use in patients perceived to be at higher risk of AKI.

Collectively, these data suggest that the mechanism of AKI is multifactorial and interdependent and seems to involve factors beyond hemodynamic disturbances including the systemic inflammatory response, free radical-induced damage, and ischemia-reperfusion injury.

\section{Limitations of the study}

Our study contains only a relatively small number of patients; therefore, certain associations may have been missed, and although the outcome of AKI was examined, the study was not powered to examine differences in temporal trend in development of AKI. Though inflammatory marker elevation parallels AKI development, etiological considerations are made with caution, and further studies are required to delineate underlying mechanisms. The association between AKI and NAC use is incidental and was not a randomized intervention in the study, so again it must be interpreted with caution.

\section{Conclusions}

Acute kidney injury after TAVI is predicted by the TA approach and absence of NAC therapy pre-procedure. TAVI generates a significant inflammatory response, with an associated increase in oxidative stress. There is also significant renal ischemia/reperfusion injury.

The changes in these processes are significantly higher after TA TAVI, which may in part explain the higher incidence of AKI in this patient cohort.

Our results inform case selection in this high-risk patient group and emphasize the importance of minimizing tissue damage and hemodynamic instability during the TAVI procedure, thus supporting the technological drive towards a minimally invasive, fully percutaneous procedure. The use of $\mathrm{NAC}$ to protect the kidneys merits further study.

\section{Acknowledgments}

The authors appreciate the work contributed by the medical and nursing staff at Kings College Hospital and the affiliated cardiology clinics.

\section{Funding}

This work was funded by King's College Hospital R\&D Grant and was supported by the
Department of Health via a National Institute for Health Research Biomedical Research Centre award to Guy's \& St. Thomas' NHS Foundation Trust in partnership with King's College London and King's College Hospital NHS Foundation Trust.

\section{Conflict of interest: None declared}

\section{References}

1. Parikh C, Coca S, Thiessen-Philbrook H, et al. Postoperative Biomarkers Predict Acute Kidney Injury and Poor Outcomes after Adult Cardiac Surgery. J Am Soc Nephrol. 2011; 22(9): 1748-1757, doi: 10.1681/asn.2010121302.

2. Genereux P, Head SJ, Wood DA, et al. Transcatheter aortic valve implantation: 10-year anniversary. Part II: clinical implications. Eur Heart J. 2012; 33(19): 2399-2402, doi: 10.1093/eurheartj/ ehs223.

3. Elhmidi Y, Bleiziffer S, Piazza N, et al. Incidence and predictors of acute kidney injury in patients undergoing transcatheter aortic valve implantation. Am Heart J. 2011; 161(4): 735-739, doi: 10.1016/j.ahj.2011.01.009, indexed in Pubmed: 21473973.

4. Van Linden A, Kempfert J, Rastan AJ. Risk of acute kidney injury after minimally invasive transapical aortic valve implantation in 270 patients. Eur J Cardiothorac Surg. 2011; 39(6): 835-842, doi: 10.1016/j.ejcts.2010.11.034, indexed in Pubmed: 21186126.

5. Nuis RJ, Van Mieghem NM, Tzikas A. Frequency, determinants, and prognostic effects of acute kidney injury and red blood cell transfusion in patients undergoing transcatheter aortic valve implantation. Catheter Cardiovasc Interv. 2011; 77(6): 881-999, doi: 10.1002/ccd.22874, indexed in Pubmed: 21061244.

6. Sinning JM, Hammerstingl C, Vasa-Nicotera M, et al. Aortic regurgitation index defines severity of peri-prosthetic regurgitation and predicts outcome in patients after transcatheter aortic valve implantation. J Am Coll Cardiol. 2012; 59(13): 1134-1141, doi: 10.1016/j.jacc.2011.11.048, indexed in Pubmed: 22440213.

7. Yong ZE, Wiegerinck EM, Boerlage van-Dijk K, et al. Transcatheter Aortic Valve Implantation. Circ Cardiovasc Intervent. 2012; 5(3): 415-423, doi: 10.1161/CIRCINTERVENTIONS.111.964882, indexed in Pubmed: 22668556.

8. Gueret G, Lion F, Guriec N, et al. Acute renal dysfunction after cardiac surgery with cardiopulmonary bypass is associated with plasmatic IL6 increase. Cytokine. 2009; 45(2): 92-98, doi: 10.1016/j.cyto.2008.11.001, indexed in Pubmed: 19128984.

9. McBride WT, Allen S, Gormley SMC, et al. Methylprednisolone favourably alters plasma and urinary cytokine homeostasis and subclinical renal injury at cardiac surgery. Cytokine. 2004; 27(2-3): 81-89, doi: 10.1016/j.cyto.2004.03.018, indexed in Pubmed: 15242697.

10. Mehta RL, Kellum JA, Shah SV, et al. Acute Kidney Injury Network: report of an initiative to improve outcomes in acute kidney injury. Crit Care. 2007; 11(2): R31, doi: 10.1186/cc5713, indexed in Pubmed: 17331245.

11. Kappetein AP, Head SJ, Généreux P, et al. Updated standardized endpoint definitions for transcatheter aortic valve implantation: the Valve Academic Research Consortium-2 consensus document. Eur Heart J. 2012; 33(19): 2403-2418, doi: 10.1093/eurheartj/ehs255, indexed in Pubmed: 23026477.

12. Dworakowski R, Wendler O, Bhan A, et al. Successful transcatheter aortic valve implantation (TAVI) is associated with transient 
left ventricular dysfunction. Heart. 2012; 98(22): 1641-1646, doi: 10.1136/heartjnl-2012-302505, indexed in Pubmed: 22914532.

13. Sinning JM, Scheer AC, Adenauer V, et al. Systemic inflammatory response syndrome predicts increased mortality in patients after transcatheter aortic valve implantation. Eur Heart J. 2012; 33(12): 1459-1468, doi: 10.1093/eurheartj/ehs002, indexed in Pubmed: 22285582.

14. Yamamoto M, Hayashida K, Mouillet G, et al. Prognostic value of chronic kidney disease after transcatheter aortic valve implantation. J Am Coll Cardiol. 2013; 62(10): 869-877, doi: 10.1016/j. jacc.2013.04.057, indexed in Pubmed: 23707321.

15. Wendler O, Maccarthy P. Renal failure after transcatheter aortic valve implantation: do we know the full story? J Am Coll Cardiol. 2013; 62(10): 878-880, doi: 10.1016/j.jacc.2013.04.058, indexed in Pubmed: 23707322.

16. Kong WY, Yong G, Irish A. Incidence, risk factors and prognosis of acute kidney injury after transcatheter aortic valve implantation. Nephrology (Carlton). 2012; 17(5): 445-451, doi: 10.1111/j.14401797.2012.01593.x, indexed in Pubmed: 22390156.

17. Stahli BE, Grunenfelder J, Jacobs S, et al. Assessment of inflammatory response to transfemoral transcatheter aortic valve implantation compared to transapical and surgical procedures: a pilot study. J Invasive Cardiol. 2012; 24(8): 407-411, indexed in Pubmed: 22865312.

18. Devarajan P. Neutrophil gelatinase-associated lipocalin: a promising biomarker for human acute kidney injury. Biomark Med. 2010; 4(2): 265-280, doi: 10.2217/bmm.10.12, indexed in Pubmed: 20406069 .

19. Koskinas KC, Stortecky S, Franzone A, et al. Post-Procedural troponin elevation and clinical outcomes following transcatheter aortic valve implantation. J Am Heart Assoc. 2016; 5(2), doi: 10.1161/JAHA.115.002430, indexed in Pubmed: 26896474.

20. Patel UD, Garg AX, Krumholz HM, et al. Preoperative serum brain natriuretic peptide and risk of acute kidney injury after cardiac surgery. Circulation. 2012; 125(11): 1347-1355, doi: 10.1161/CIRCULATIONAHA.111.029686, indexed in Pubmed: 22322531.

21. Jeong EuG, Nam HS, Lee SuMi, et al. Role of B-type natriuretic peptide as a marker of mortality in acute kidney injury patients treated with continuous renal replacement therapy. Ren Fail. 2013; 35(9): 1216-1222, doi: 10.3109/0886022X.2013.823870, indexed in Pubmed: 23924312.

22. Dworakowski R, Wendler O. Optimal pain management after aortic valve implantation: an opportunity to improve outcomes after transapical access in the future? Heart. 2012; 98(21): 1541-1542, doi: 10.1136/heartjnl-2012-302876, indexed in Pubmed: 22976201.

23. McCullough PA, Duc P, Omland T, et al. B-type natriuretic peptide and renal function in the diagnosis of heart failure: an analysis from the Breathing Not Properly Multinational Study. Am J Kidney Dis. 2003; 41(3): 571-579, doi: 10.1053/ajkd.2003.50118, indexed in Pubmed: 12612980.

24. Vickery S, Price CP, John RI, et al. B-type natriuretic peptide (BNP) and amino-terminal proBNP in patients with CKD: relationship to renal function and left ventricular hypertro- phy. Am J Kidney Dis. 2005; 46(4): 610-620, doi: 10.1053/j. ajkd.2005.06.017, indexed in Pubmed: 16183415.

25. Takami Y, Horio T, Iwashima Y, et al. Diagnostic and prognostic value of plasma brain natriuretic peptide in non-dialysis-dependent CRF. Am J Kidney Diseases. 2004; 44(3): 420-428, doi: 10.1016/s0272-6386(04)00812-1.

26. Weinberg EO, Shimpo M, Hurwitz S, et al. Identification of serum soluble ST2 receptor as a novel heart failure biomarker. Circulation. 2003; 107(5): 721-726, doi: 10.1161/01. cir.0000047274.66749.fe, indexed in Pubmed: 12578875.

27. Weir RA, Miller AM, Murphy GE, et al. Serum soluble ST2: a potential novel mediator in left ventricular and infarct remodeling after acute myocardial infarction. J Am Coll Cardiol. 2010; 55(3): 243-250, doi: 10.1016/j.jacc.2009.08.047, indexed in Pubmed: 20117403.

28. Sabatine MS, Morrow DA, Higgins LJ, et al. Complementary roles for biomarkers of biomechanical strain ST2 and N-terminal prohormone B-type natriuretic peptide in patients with ST-elevation myocardial infarction. Circulation. 2008; 117(15): 1936-1944, doi: 10.1161/CIRCULATIONAHA.107.728022, indexed in Pubmed: 18378613.

29. Akcay A, He Z, et al. IL-33 exacerbates acute kidney injury. J Am Soc Nephrol. 2011; 22(11): 2057-2067, doi: 10.1681/ ASN.2010091011, indexed in Pubmed: 21949094.

30. Kodali S, Williams M, Smith C, et al. Two-Year Outcomes after Transcatheter or Surgical Aortic-Valve Replacement. N Engl J Med. 2012; 366(18): 1686-1695, doi: 10.1056/NEJMoa1200384, indexed in Pubmed: 22443479.

31. Athappan G, Patvardhan E, Tuzcu E, et al. Incidence, predictors, and outcomes of aortic regurgitation after transcatheter aortic valve replacement. J Am Coll Cardiol. 2013; 61(15): 1585-1595, doi: 10.1016/j.jacc.2013.01.047.

32. Dworakowski R, Wendler O, Halliday B, et al. Device-dependent association between paravalvar aortic regurgitation and outcome after TAVI. Heart. 2014; 100(24): 1939-1945, doi: 10.1136/ heartjnl-2013-305390.

33. Tepel M, Giet Mv, Schwarzfeld C, et al. Prevention of radiographic-contrast-agent-induced reductions in renal function by acetylcysteine. N Engl J Med. 2000; 343(3): 180-184, doi: 10.1056/ nejm200007203430304.

34. Aruoma O, Halliwell B, Hoey B, et al. The antioxidant action of $\mathrm{N}$-acetylcysteine: Its reaction with hydrogen peroxide, hydroxyl radical, superoxide, and hypochlorous acid. Free Radic Biol Med. 1989; 6(6): 593-597, doi: 10.1016/0891-5849(89)90066-x.

35. Fishbane S. N-acetylcysteine in the prevention of radiocontrast-induced nephropathy. J Am Society Nephrol. 2004; 15(2): 251-260, doi: 10.1097/01.asn.0000107562.68920.92.

36. Nitescu N, Ricksten SE, Marcussen N, et al. N-acetylcysteine attenuates kidney injury in rats subjected to renal ischaemia-reperfusion. Nephrology Dialysis Transplantation. 2006; 21(5): 1240-1247, doi: 10.1093/ndt/gfk032.

37. Patel NN, Rogers CA, Angelini GD, et al. Pharmacological therapies for the prevention of acute kidney injury following cardiac surgery: a systematic review. Heart Fail Rev. 2011; 16(6): 553-567, doi: 10.1007/s10741-011-9235-5, indexed in Pubmed: 21400231. 